\title{
Interaction-driven Band Flattening and Correlated Phases in Twisted Bilayer Graphene
}

Youngjoon Choi ${ }^{1,2,3 *}$, Hyunjin Kim ${ }^{1,2,3 *}$, Cyprian Lewandowski ${ }^{2,3,4}$, Yang Peng ${ }^{5}$, Alex Thomson ${ }^{2,3,4}$, Robert Polski $^{1,2}$, Yiran Zhang ${ }^{1,2,3}$, Kenji Watanabe ${ }^{6}$, Takashi Taniguchi ${ }^{6}$, Jason Alicea ${ }^{2,3,4}$, Stevan Nadj-Perge ${ }^{1,2 \dagger}$

${ }^{1}$ T. J. Watson Laboratory of Applied Physics, California Institute of Technology, 1200 East California Boulevard, Pasadena, California 91125, USA

${ }^{2}$ Institute for Quantum Information and Matter, California Institute of Technology, Pasadena, California 91125, USA

${ }^{3}$ Department of Physics, California Institute of Technology, Pasadena, California 91125, USA

${ }^{4}$ Walter Burke Institute for Theoretical Physics, California Institute of Technology, Pasadena, California 91125, USA

${ }^{5}$ Department of Physics and Astronomy, California State University, Northridge, California 91330, USA

${ }^{6}$ National Institute for Materials Science, Namiki 1-1, Tsukuba, Ibaraki 305 0044, Japan

*These authors contributed equally to this work

†Correspondence: s.nadj-perge@caltech.edu

Flat electronic bands, characteristic of magic-angle twisted bilayer graphene (TBG), host a wealth of correlated phenomena. Early theoretical considerations ${ }^{1 / 2}$ suggested that, at the magic angle, the Dirac velocity vanishes and the entire width of the moiré bands becomes extremely narrow. Yet, this scenario contradicts experimental studies ${ }^{3}-11$ that reveal a finite Dirac velocity as well as bandwidths significantly larger than predicted. Although more sophisticated modeling 12 can, in part, account for the bandwidth broadening, many essential aspects of magic-angle TBG bands and emerging correlated phenomena remain elusive. Here we use spatially resolved spectroscopy in finite and zero magnetic fields to examine the electronic structure of moiré bands and their intricate connection to correlated phases. By following the relative shifts of Landau levels in finite fields, we detect filling-dependent band flattening caused by strong interactions between electrons, that unexpectedly starts already at $\sim 1.3^{\circ}$, well above the magic angle and hence nominally in the weakly correlated regime. We further show that, as the twist angle is reduced, the moiré bands become maximally flat at progressively lower doping levels. Surprisingly, when the twist angles reach values for which the maximal flattening occurs at approximate filling of $-2,+1,+2,+3$ electrons per moiré unit cell, the corresponding zero-field correlated phases start to emerge. Our observations are corroborated by calculations that incorporate an interplay between the Coulomb charging energy and exchange interactions; together these effects produce band flattening and hence a significant density-of-states enhancement that facilitates the observed symmetrybreaking cascade transitions. Besides emerging phases pinned to integer fillings, we also experimentally identify a series of pronounced correlation-driven band deformations and soft gaps in a wider doping range around \pm 2 filling where superconductivity is expected. Our 
results highlight the essential role of interaction-driven band-flattening in defining electronic properties and forming robust correlated phases in TBG.

Figure 1 1a sketches our scanning tunneling microscopy (STM) setup. TBG is placed on an atomically smooth dielectric consisting of monolayer $\mathrm{WSe}_{2}$ and a thicker $(\sim 30 \mathrm{~nm})$ layer hexagonal boron nitride $(\mathrm{hBN})^{\sqrt{10}}$ (see also Supplementary Information (SI), section 1). By applying a gate voltage $V_{\text {Gate }}$ on a graphite gate underneath, we tune the TBG charge density, or equivalently filling factor $\nu$ corresponding to the number of electrons per moiré unit cell. Typical TBG topography shows a moiré superlattice consisting of AA sites, where the local density of states (LDOS) originating from bands closest to the Fermi energy at charge neutrality is predominantly concentrated $^{4-7}$, and AB sites in between (Fig. 1 17). Local twist angle and strain are determined by measuring distances between neighboring AA sites ${ }^{45}$ (see also SI, section 2). We first focus on a TBG region with local twist angle $1.32^{\circ}$ to show that interactions play an important role even well above the magic angle of $\sim 1.1^{\circ}$.

To further examine the moiré band structure, we probe Landau levels (LLs) that develop when an out-of-plane magnetic field is applied. The tunneling conductance spectrum taken on an AB site shows two different sets of LLs observed as LDOS peaks separated by the VHSs (Fig. 17, d). The LLs from the inner set, with energies bounded within the two VHSs 9.10 , originate from band pockets around the $\kappa$ and $\kappa^{\prime}$ high symmetry points of the moiré Brillouin zone; we therefore denote them as $\kappa$ LLs (see Fig. 11). Similarly we define LLs from the outer set as $\gamma$ LLs since they originate from portions of the bands around the $\gamma$ point. This assignment is justified by the magnetic-field dependence of the observed LL spectrum (see also Supplementary Fig. S1). In particular, upon increasing the magnetic field the zeroth $\gamma \mathrm{LLs}\left(\gamma \mathrm{LL}_{0}\right.$ in the valance and conduction bands) approach the VHSs, as expected from the conduction- and valence-band dispersion at the $\gamma$ point; the $\kappa$ LL energies, in contrast, do not change-consistent with the zeroth LLs expected from the Dirac-like dispersion at the $\kappa, \kappa^{\prime}$ points. Moreover, even though both $\kappa$ LLs and $\gamma$ LLs are visible on the AB sites, only the $\kappa$ LLs are resolved on AA sites (Supplementary Fig. S2a, b). This observation suggests that the spectral weight of the $\kappa, \kappa^{\prime}$ pockets is spatially located predominantly on AA sites while the weight of the $\gamma$ pocket is more distributed over AB sites and domain walls, in line with previous theoretical calculations $17+19$.

Importantly, the energy separation between $\gamma \mathrm{LL}_{0}$ and $\gamma \mathrm{LL}_{1}$ changes with carrier density (Fig. 1c, d), signaling significant deformation of the moiré bands upon doping even at the large $1.32^{\circ}$ twist angle. For the conduction band, the separation is maximized at $\nu=-4$, where $E\left(\gamma \mathrm{LL}_{0}\right)-E\left(\gamma \mathrm{LL}_{1}\right) \approx 25 \mathrm{meV}$, and monotonically decreases below $5 \mathrm{meV}$ near $\nu=+4$ (Fig. $1 \mathrm{~g}$ ). For the valence band, the trend is reversed: the separation between $\gamma$ LLs increases with filling factor (see Supplementary Fig. S3 3 , b, on remote bands and extracted effective mass). Note that a displacement field, likely present due to the single back-gate geometry of our device, might also slightly modify the band structure with doping. However, the displacement field would change the conduction and the valence bands symmetrically, in contrast to the observed asymmetric evolution of $\gamma$ LLs (Supplementary Fig. S3r, d and SI, sections 3, 4 and 5). 
The relative $\gamma$ LL shifts upon doping are well-captured within a model that includes interaction effects deriving from the inhomogeneous charge distribution in a moiré unit cell ${ }^{18}[20-23$. Starting from charge neutrality, electrons are first added or removed from states near $\kappa, \kappa^{\prime}$ that localize primarily on AA sites-creating an associated inhomogeneous electrostatic Hartree potential peaked in the AA regions. States near the $\gamma$ point feel this potential less strongly because they are relatively delocalized within the unit cell, which in turn renormalizes the energy cost for populating states near $\gamma$. More generally, each part of the moiré bands experiences a different electrostatic potential, creating filling-dependent band deformations. Figure 1e presents the calculated band structures for different integer fillings at $\mathrm{B}=0 \mathrm{~T}$ (see SI, section 3 for details of the calculation). The conduction and valence bands deform asymmetrically upon doping: the conduction (valence) band becomes flatter and the valence (conduction) band more dispersive as the filling factor increases (decreases). Consequently, in finite magnetic fields the energy separation between $\gamma$ LLs also changes asymmetrically (Fig. $1 \mathrm{~g}$ inset). The Landau level spectrum evaluated with only the electrostatic Hartree potential (Fig. 1f; see also SI, section 5) indeed reproduces the main features of the experimental data.

Interaction-induced deformation of the moiré bands completely flattens the $\gamma$ pocket when the twist angle approaches the magic-angle value, as can be deduced from the measured evolution of $\gamma$ LLs (Fig. 2). To explore the twist-angle dependence, we focus on an area where the angle changes over a $\sim 600 \mathrm{~nm}$ area and strain is relatively low $(<0.3 \%)$; see Fig. 2a. Spatially resolved measurements in this area reveal the twist angle-dependent evolution of LLs with electrostatic doping (Fig. 2p-e). At $\mathrm{V}_{\text {Gate }}$ near the charge neutrality point (CNP) (Fig. 2p), we observe that $\gamma \mathrm{LL}_{0}$, for both the valence and conduction moiré bands, eventually merges with the corresponding VHS - signaling maximal band flattening. The onset of merging occurs at somewhat larger angles for the valence band $\left(\sim 1.15^{\circ}\right)$ compared to the conduction band $\left(\sim 1.1^{\circ}\right)$. Moreover, doping strongly shifts this onset. For example, in the valence band (Fig. 25-e) the maximal band flattening moves considerably towards larger (smaller) twist angles for hole (electron) doping (see Supplementary Fig. S4 for the conduction band).

More detailed examples of the evolution of LLs, along with the development of correlated gaps in finite fields, can be seen in the doping vs. bias maps of Fig. 2 f-h (See also Supplementary Fig. S5 for more data). At $1.23^{\circ}$ (Fig. 2f), the $\gamma \mathrm{LL}_{0}$ energies are well-resolved in both the valence and conduction bands between $\nu \approx-2.2$ and $\nu \approx+3.3$, beyond which one of the two merges with the corresponding VHS (and also Fermi energy $V_{\text {Bias }}=0 \mathrm{mV}$; see discussion of Fig. 3 below). The difference in filling factors of the merging points for the conduction and valence bands reflects an appreciable electron-hole asymmetry. At this large twist angle, aside from quantum Hall ferromagnetism in the zeroth $\kappa \mathrm{LL}$ (responsible for the structure between $\nu=-1$ and +1 ), no pronounced correlated gaps are observed at the Fermi energy. As the twist angle is decreased to $1.17^{\circ}, \gamma \mathrm{LL}_{0}$ in the valence band merges at a lower $|\nu|$, and an additional correlated gap appears when the merged $\gamma \mathrm{LL}_{0}$ crosses the Fermi energy (black arrow in Fig. $2 \mathrm{~g}$ ). For an even lower angle of $1.15^{\circ}$, correlated gaps also begin to emerge on the electron-doped side after $\gamma \mathrm{LL}_{0}$ is merged with conductance band VHS (black arrows in Fig. 2h). These correlated gaps correspond to Chern 
insulators that emanate from integer filling factors when electron-electron interactions are strong

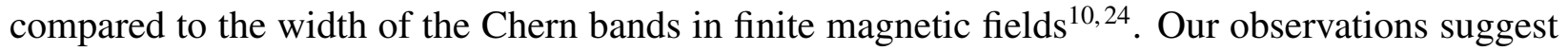
that such correlated phases emerge only once portions of the bands around $\gamma$ become maximally flat and join with the VHS.

To explore the development of zero-field correlated phases as a function of twist angle, we perform angle-dependent gate spectroscopy to trace out the evolution of the LDOS at the Fermi level10 $\left(V_{\text {Bias }} \approx 0\right)$ as a function of charge density $\left(V_{\text {Gate }}\right)$; see Fig. 3a-b. Pronounced LDOS suppression near the Fermi energy occurs at certain integer filling factors $\nu$. For all angles, prominent suppression is observed at $\nu= \pm 4,0$, reflecting the small LDOS around the CNP $(\nu=0)$ and band gaps at full fillings $(\nu= \pm 4)$. At $\nu= \pm 2,+3,+1$ we additionally observe sharp LDOS drops that can be attributed to emerging correlated gaps similar to those resolved in transpor ${ }^{3 / 25}$ 27. Importantly, the observed LDOS suppressions at integer fillings begin to emerge within the same range of angles that displayed considerable band flattening in finite fields (Fig. 2, Fig. 3 a and Supplementary Fig. S6. The angle onsets of the insulating regions for the conduction and valence bands (marked by dashed lines and arrows) have an electron-hole asymmetry that is also consistent with the band flattening. Spectra at $\nu= \pm 2$ taken at various twist angles (Fig. 3k,d) indeed show that LDOS suppressions originate from the development of a gap at the Fermi energy, and that the $\nu=-2$ gap emerges at slightly higher angles-corroborating electron-hole asymmetry. The maximal size of this half-filling gap is $\sim 1.5 \mathrm{meV}$, lower than the initial reports from spectroscopic measurements ${ }^{4 / 6}$, but slightly larger than the activation gap extracted from transpor ${ }^{3 / 25}$. 27 . We note that the LDOS suppression from the observed gaps in Fig. $3 \mathrm{k}$,d may also be, in part, related to the Fermi surface reconstruction due to flavour symmetry-breaking cascade ${ }^{28129}$ (see also discussion of Fig. 47. As we show in the following, the band flattening also creates conditions that favor the cascade.

A theory analysis of the continuum-model band structure $\frac{1 \mid 130}{13}$ with interactions treated at a mean-field level in part accounts for the observed band flattening and related symmetry-breaking cascade instabilities near the magic angle (see SI, section 6 for details). While the doping dependence of the moiré band deformation at larger angles is well-modeled by assuming only a Hartree correction (Fig. 1), near the magic angle a more complete Hartree-Fock treatment is necessary. For example, including only the Hartree term ${ }^{18|20| 22}$ predicts a dramatic band inversion at the $\gamma$ pocket that is not observed experimentally (Supplementary Fig. S10 -d). The Fock term in part counteracts this band inversion - thereby stabilizing the band flattening; see Fig. 3e-g, Supplementary Fig. S10 -h and SI, section 3. Importantly, our calculations predict that near the magic angle, the density of states (DOS) at the Fermi level $\left(\mathrm{E}_{\mathrm{F}}\right)$ is significantly amplified (by up to a factor of $\sim 4$ for $\nu=2$ and $\sim 15$ for $\nu=3$ ) relative to expectations from non-interacting models (Fig. 3h). This considerable interaction-driven DOS increase magnifies the already high DOS around the VHS and accordingly promotes the symmetry-breaking cascade of electronic transitions ${ }^{28}$. For a more quantitative treatment of the cascade instabilities, we compare the energies of unpolarized and flavor-polarized states at integer fillings for different twist angles. In a non-self-consistent treatment that neglects band renormalization (see Supplementary Fig. S10, depending on the model, 
either no cascade is expected, or flavor polarization is preferred only in a narrow window around the magic angle. A self-consistent treatment that incorporates band flattening (Fig. 3), by contrast, predicts cascading over broader twist-angle windows whose widths depend strongly on filling-in agreement with experimental data on the electron-doped side of Fig. 3 a (see also Supplementary Fig. S9].

Our observations reconcile the apparent discrepancy between the emergence of correlated phases around the magic angle and experimental observations at odds with seemingly crucial aspects of the band theory predictions: (i) large Dirac velocity around the CNP ${ }^{3 / 10}$, (ii) total bandwidth exceeding $40 \mathrm{meV}^{4}{ }^{828}$, and (iii) large separation of the VHSs ${ }^{4.7}$. These quantities, Dirac velocity, total bandwidth and the VHS separation, appear not to be essential; for example Supplementary Fig. S7 shows that measured Dirac velocity and VHS separation are even smaller at $1.18^{\circ}$ than $1.04^{\circ}$, while signatures of strongly correlated phases (e.g. symmetry-breaking cascade and correlated gaps) are only present in $1.04^{\circ}$ comparing these two angles. Instead, we identify the interaction-driven flattening of the moiré bands around the $\gamma$ pockets, with the consequent increase in the density of states, as the decisive feature needed for the formation of correlated phases.

In addition to correlated gaps pinned to integer fillings $\nu= \pm 2,+1,+3$, we also observe several other interaction-driven features near the magic angle that have not been discussed in previous STM measurements (Fig. 4). For example, prominent LDOS suppressions at the Fermi level are visible in both the $2<\nu<3$ and $-3<\nu<-2$ doping regions (Fig. $4 \mathrm{~b}-\mathrm{d}$ ). Of the two regions, the feature between $\nu=+2$ and +3 has a 'tilted $\mathrm{V}$ ' shape at small bias voltages, which can be largely understood as a consequence of the relative prominence of the flavour polarization in the conduction band compared to the valence band ${ }^{10}$ (see also Fig. 3 a-d). There, two of the four flavors are pushed away from the Fermi energy by strong interactions ${ }^{28}$, consistent with the LDOS being predominately shifted to higher energy (Fig. $4 \mathrm{a}, \mathrm{c}$ ) and resulting in a highly asymmetric spectrum, as seen in compressibility measurements ${ }^{28}$. In contrast, the spectrum between $\nu=-2$ and -3 exhibits a slightly different shape that can not be fully explained by a simple flavoursymmetry-breaking cascade that produces large overall asymmetry around the Fermi energy. Additionally, this doping range also shows a clear, more symmetric gapped feature at small bias voltages (Fig. 4e). The corresponding gap, spanning almost the entire filling range $-3<\nu<-2$ (Fig. 4d), reaches its maximal size of $2 \Delta \approx 1.1 \mathrm{meV}$. Also, it becomes prominent only below $\theta=1.16^{\circ}$ (see Supplementary Fig. S6), and gradually recedes with temperature and disappears above $5-7$ K (Fig. 4e). The gap size as well the temperature dependence of this feature is similar to the gap at $\nu=-2$; however, the filling range observed here is unusually large and cannot be explained simply by non-interacting effects (see Supplementary Fig. S8). Furthermore, the gap extends over a filling range comparable to that at which superconductivity has been observed in transport at similar angles for TBG on $\mathrm{WSe}_{2}{ }^{27}$ as well as in many $\mathrm{hBN}$-only encapsulated devices $25,26,31$. This correspondence indicates that the observed feature may be related to superconductivity itself or signals the possible existence of a pseudo-gap phase that precedes superconductivity ${ }^{\sqrt{2}}$. Regardless of its exact origin, the pronounced suppression (instead of increase) of the LDOS near the Fermi energy, together with symmetry-breaking cascade features, for fillings where superconductivity is 
anticipated may suggest either an electronic pairing origin ${ }^{33}$ or a regime of strong-coupling superconductivity as recently pointed out for magic-angle trilayer graphene ${ }^{34} 35$. Another interesting observation is that, while the gap-like features at the Fermi energy become weaker with increasing temperature, features at higher energies-previously identified to be related to symmetry-breaking cascade transitions 9 10 29 — are enhanced, see the temperature evolution in Fig. 4 $\mathrm{f}-\mathrm{i}$. The relation of these features and various recently reported phases that emerge at elevated temperatures $36 \mathbf{3 7}$ remains a subject for future investigations.

\section{References:}

1. Bistritzer, R. \& MacDonald, A. H. Moiré bands in twisted double-layer graphene. Proceedings of the National Academy of Sciences 108, 12233-12237 (2011).

2. Lopes dos Santos, J. M. B., Peres, N. M. R. \& Castro Neto, A. H. Graphene Bilayer with a Twist: Electronic Structure. Physical Review Letters 99, 256802 (2007).

3. Cao, Y. et al. Correlated insulator behaviour at half-filling in magic-angle graphene superlattices. Nature 556, 80-84 (2018).

4. Kerelsky, A. et al. Maximized electron interactions at the magic angle in twisted bilayer graphene. Nature 572, 95-100 (2019).

5. Choi, Y. et al. Electronic correlations in twisted bilayer graphene near the magic angle. Nature Physics 15, 1174-1180 (2019).

6. Xie, Y. et al. Spectroscopic signatures of many-body correlations in magic-angle twisted bilayer graphene. Nature 572, 101-105 (2019).

7. Jiang, Y. et al. Charge order and broken rotational symmetry in magic-angle twisted bilayer graphene. Nature 573, 91-95 (2019).

8. Tomarken, S. L. et al. Electronic Compressibility of Magic-Angle Graphene Superlattices. Physical Review Letters 123, 046601 (2019).

9. Nuckolls, K. P. et al. Strongly correlated Chern insulators in magic-angle twisted bilayer graphene. Nature 588, 610-615 (2020).

10. Choi, Y. et al. Correlation-driven topological phases in magic-angle twisted bilayer graphene. Nature 1-6 (2021).

11. Park, J. M., Cao, Y., Watanabe, K., Taniguchi, T. \& Jarillo-Herrero, P. Flavour Hund's Coupling, Correlated Chern Gaps, and Diffusivity in Moir \'e Flat Bands. arXiv:2008.12296 [cond-mat] (2020). 2008.12296.

12. Uchida, K., Furuya, S., Iwata, J.-I. \& Oshiyama, A. Atomic corrugation and electron localization due to Moir \'e patterns in twisted bilayer graphenes. Physical Review B 90, 155451 (2014). 
13. Jung, J., Raoux, A., Qiao, Z. \& MacDonald, A. H. Ab initio theory of moir $\backslash$ 'e superlattice bands in layered two-dimensional materials. Physical Review B 89, 205414 (2014).

14. Nam, N. N. T. \& Koshino, M. Lattice relaxation and energy band modulation in twisted bilayer graphene. Physical Review B 96, 075311 (2017).

15. Carr, S., Fang, S., Zhu, Z. \& Kaxiras, E. Exact continuum model for low-energy electronic states of twisted bilayer graphene. Physical Review Research 1, 013001 (2019).

16. Guinea, F. \& Walet, N. R. Continuum models for twisted bilayer graphene: Effect of lattice deformation and hopping parameters. Physical Review B 99, 205134 (2019).

17. Rademaker, L. \& Mellado, P. Charge-transfer insulation in twisted bilayer graphene. Physical Review B 98, 235158 (2018).

18. Guinea, F. \& Walet, N. R. Electrostatic effects, band distortions, and superconductivity in twisted graphene bilayers. Proceedings of the National Academy of Sciences 115, 1317413179 (2018).

19. Carr, S., Fang, S., Po, H. C., Vishwanath, A. \& Kaxiras, E. Derivation of Wannier orbitals and minimal-basis tight-binding Hamiltonians for twisted bilayer graphene: First-principles approach. Physical Review Research 1, 033072 (2019).

20. Rademaker, L., Abanin, D. A. \& Mellado, P. Charge smoothening and band flattening due to Hartree corrections in twisted bilayer graphene. Physical Review B 100, 205114 (2019).

21. Cea, T., Walet, N. R. \& Guinea, F. Electronic band structure and pinning of Fermi energy to Van Hove singularities in twisted bilayer graphene: A self-consistent approach. Physical Review B 100, 205113 (2019).

22. Goodwin, Z. A. H., Vitale, V., Liang, X., Mostofi, A. A. \& Lischner, J. Hartree theory calculations of quasiparticle properties in twisted bilayer graphene. arXiv:2004.14784 [cond-mat] (2020). 2004.14784.

23. Calderón, M. J. \& Bascones, E. Interactions in the 8-orbital model for twisted bilayer graphene. arXiv:2007.16051 [cond-mat] (2020). 2007.16051.

24. Saito, Y. et al. Hofstadter subband ferromagnetism and symmetry broken Chern insulators in twisted bilayer graphene. arXiv:2007.06115 [cond-mat] (2020). 2007.06115.

25. Yankowitz, M. et al. Tuning superconductivity in twisted bilayer graphene. Science 363, 1059-1064 (2019).

26. Lu, X. et al. Superconductors, orbital magnets and correlated states in magic-angle bilayer graphene. Nature 574, 653-657 (2019).

27. Arora, H. S. et al. Superconductivity in metallic twisted bilayer graphene stabilized by WSe 2. Nature 583, 379-384 (2020). 
28. Zondiner, U. et al. Cascade of phase transitions and Dirac revivals in magic-angle graphene. Nature 582, 203-208 (2020). 1912 .06150.

29. Wong, D. et al. Cascade of transitions between the correlated electronic states of magic-angle twisted bilayer graphene. arXiv:1912.06145 [cond-mat] (2019). 1912.06145.

30. Koshino, M. et al. Maximally Localized Wannier Orbitals and the Extended Hubbard Model for Twisted Bilayer Graphene. Physical Review X 8, 031087 (2018).

31. Cao, Y. et al. Unconventional superconductivity in magic-angle graphene superlattices. Nature 556, 43-50 (2018).

32. Hofmann, J. S., Berg, E. \& Chowdhury, D. Superconductivity, pseudogap, and phase separation in topological flat bands. Physical Review B 102, 201112 (2020).

33. Bernevig, B. A. et al. TBG V: Exact Analytic Many-Body Excitations In Twisted Bilayer Graphene Coulomb Hamiltonians: Charge Gap, Goldstone Modes and Absence of Cooper Pairing. arXiv:2009.14200 [cond-mat] (2020). 2009.14200.

34. Park, J. M., Cao, Y., Watanabe, K., Taniguchi, T. \& Jarillo-Herrero, P. Tunable Phase Boundaries and Ultra-Strong Coupling Superconductivity in Mirror Symmetric Magic-Angle Trilayer Graphene. arXiv:2012.01434 [cond-mat] (2020). 2012.01434.

35. Hao, Z. et al. Electric field tunable unconventional superconductivity in alternating twist magic-angle trilayer graphene. arXiv:2012.02773 [cond-mat] (2020). 2012.02773.

36. Saito, Y. et al. Isospin Pomeranchuk effect and the entropy of collective excitations in twisted bilayer graphene. arXiv:2008.10830 [cond-mat] (2020). 2008.10830.

37. Rozen, A. et al. Entropic evidence for a Pomeranchuk effect in magic angle graphene. arXiv:2009.01836 [cond-mat] (2020). 2009.01836.

38. Walkup, D. et al. Tuning single-electron charging and interactions between compressible Landau level islands in graphene. Physical Review B 101, 035428 (2020).

39. Cea, T. \& Guinea, F. Band structure and insulating states driven by Coulomb interaction in twisted bilayer graphene. Physical Review B 102, 045107 (2020).

40. Xie, M. \& MacDonald, A. H. Weak-field Hall Resistivity and Spin/Valley Flavor Symmetry Breaking in MAtBG. arXiv:2010.07928 [cond-mat] (2020). 2010.07928.

41. Bultinck, N. et al. Ground State and Hidden Symmetry of Magic-Angle Graphene at Even Integer Filling. Physical Review X 10, 031034 (2020).

42. Khalaf, E., Chatterjee, S., Bultinck, N., Zaletel, M. P. \& Vishwanath, A. Charged Skyrmions and Topological Origin of Superconductivity in Magic Angle Graphene. arXiv:2004.00638 [cond-mat] (2020). 2004.00638 . 
43. Xie, M. \& MacDonald, A. H. Nature of the Correlated Insulator States in Twisted Bilayer Graphene. Physical Review Letters 124, 097601 (2020).

44. Fang, S., Carr, S., Zhu, Z., Massatt, D. \& Kaxiras, E. Angle-Dependent $\{\backslash$ it Ab initio\} Low-Energy Hamiltonians for a Relaxed Twisted Bilayer Graphene Heterostructure. 1908.00058[cond.mat] (2019).

45. Goodwin, Z. A. H., Corsetti, F., Mostofi, A. A. \& Lischner, J. Attractive electron-electron interactions from internal screening in magic-angle twisted bilayer graphene. Physical Review B 100, 235424 (2019).

46. Bistritzer, R. \& MacDonald, A. H. Moir \'e butterflies in twisted bilayer graphene. Physical Review B 84, 035440 (2011).

47. Hejazi, K., Liu, C. \& Balents, L. Landau levels in twisted bilayer graphene and semiclassical orbits. Physical Review B 100, 035115 (2019).

48. Klebl, L., Goodwin, Z. A. H., Mostofi, A. A., Kennes, D. M. \& Lischner, J. Importance of long-ranged electron-electron interactions for the magnetic phase diagram of twisted bilayer graphene. ArXiv:2012.14499[cond.mat] (2020).

49. Efros, A. L. Coulomb gap in disordered systems. Journal of Physics C: Solid State Physics 9 , 2021-2030 (1976).

50. Jung, S. et al. Evolution of microscopic localization in graphene in a magnetic field from scattering resonances to quantum dots. Nature Physics 7, 245-251 (2011).

Acknowledgments: We acknowledge discussions with Francisco Guinea, Felix von Oppen, and Gil Refael. Funding: This work has been primarily supported by NSF grants DMR-2005129 and DMR-172336; and Army Research Office under Grant Award W911NF17-1-0323. Part of the STM characterization has been supported by NSF CAREER program (DMR-1753306). Nanofabrication efforts have been in part supported by DOE-QIS program (DE-SC0019166). S.N-P. acknowledges support from the Sloan Foundation. J.A. and S.N.-P. also acknowledge support of the Institute for Quantum Information and Matter, an NSF Physics Frontiers Center with support of the Gordon and Betty Moore Foundation through Grant GBMF1250; C.L. acknowledges support from the Gordon and Betty Moore Foundation's EPiQS Initiative, Grant GBMF8682. A.T. and J.A. are grateful for the support of the Walter Burke Institute for Theoretical Physics at Caltech. Y.P. acknowledges support from the startup fund from California State University, Northridge. Y.C. and H.K. acknowledge support from the Kwanjeong fellowship.

Author Contribution: Y.C. and H.K. fabricated samples with the help of R.P. and Y.Z., and performed STM measurements. Y.C., H.K., and S.N.-P. analyzed the data. C.L. and Y.P. implemented TBG models. C.L., Y.P., and A.T. provided theoretical analysis of the model results supervised 
by J.A. S.N.-P. supervised the project. Y.C., H.K., C.L., Y.P., A.T., J.A., and S.N.-P. wrote the manuscript with input from other authors.

Data availability: The data that support the findings of this study are available from the corresponding authors on reasonable request. 

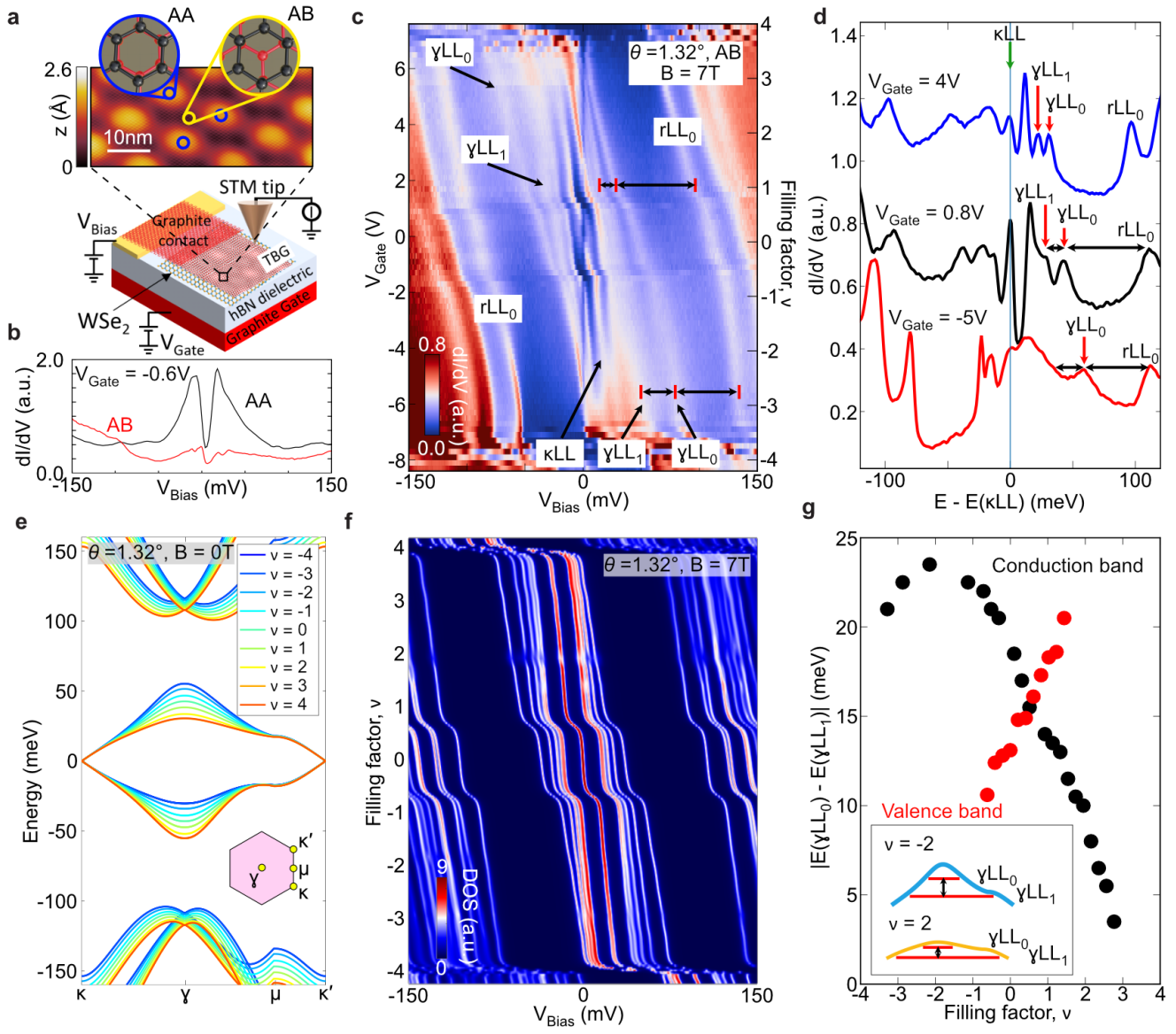

Figure $1 \mid$ Filling-dependent band structure deformation of TBG at twist angle $\theta=1.32^{\circ}$. a, Device schematics and a TBG surface topography. TBG is placed on a monolayer $\mathrm{WSe}_{2}$, thin $\mathrm{hBN}$ layer and graphite back gate. A bias voltage $\mathrm{V}_{\text {Bias }}$ is applied through a graphite contact placed on top. Blue and yellow circles respectively indicate AA- and AB/BA-stacked regions in the TBG moiré pattern (tunneling set point parameters: $V_{\text {Bias }}=100 \mathrm{mV}, \mathrm{I}=20 \mathrm{pA}$ ). b, Point spectroscopy at $\mathrm{B}=0 \mathrm{~T}$ near the CNP taken at an AA and an AB site; AA sites show large LDOS peaks corresponding to VHSs. c, Tunneling conductance (dI/dV) spectroscopy on an AB site as a function of $V_{\text {Gate }}$ at a magnetic field of $B=7 \mathrm{~T}(\mathrm{~T}=2 \mathrm{~K})$ showing the evolution of LLs with electrostatic doping. The LLs originating from $\gamma$ and $\kappa$ pockets ( $\gamma$ LLs and $\kappa$ LLs) of the flat bands as well LLs from remote bands ( $r$ LLs) are identified. The energy separation between different LLs, as marked by black lines, changes with $V_{\text {Gate }}$. See SI, section 2, for conversion between $V_{\text {Gate }}$ and $\nu$. d, Linecuts of data in (c) at $\mathrm{V}_{\text {Gate }}=4 \mathrm{~V}, 1 \mathrm{~V},-5 \mathrm{~V}$ further illustrate the LL spectrum and its change with electrostatic doping. e, Calculated TBG band structure with Hartree corrections for $\theta=1.32^{\circ}$ and $\mathrm{B}=0 \mathrm{~T}$. Electron doping flattens the conduction band while hole doping flattens the valence band. $\mathbf{f}$, Calculated density of states with Hartree corrections as a function of filling for $\mathrm{B}=7 \mathrm{~T}$ (see SI, sections 4 and 5). g, Measured energy separation between $\gamma \mathrm{LL}_{0}$ and $\gamma \mathrm{LL}_{1}$ as a function of filling factor showing conductance (valence) band flattening for electron (hole) doping. 


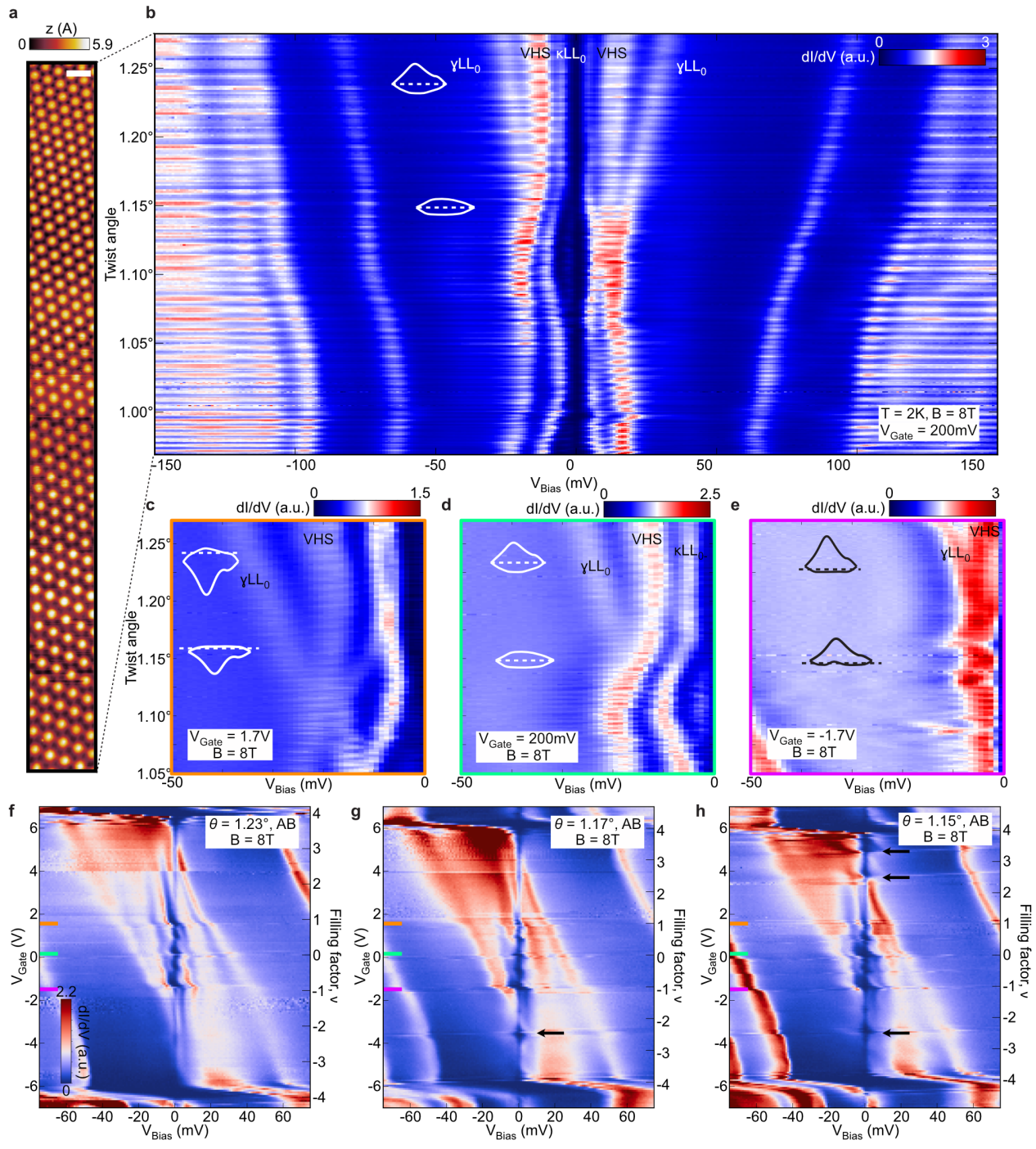

Figure $2 \mid$ Evolution of LLs with twist angle and correlated gaps at B $=8$ T. a, A $590 \mathrm{~nm} \times 50$ $\mathrm{nm}$ area where twist angle slowly changes from $1.27^{\circ}$ to $0.97^{\circ}$ (scale bar corresponds to $20 \mathrm{~nm}$, set point parameters: $\left.\mathrm{V}_{\text {Bias }}=100 \mathrm{mV}, \mathrm{I}=20 \mathrm{pA}\right)$. b, spectroscopic map near $\mathrm{CNP}\left(\mathrm{V}_{\text {Gate }}=0.2 \mathrm{~V}\right)$ taken over the same area, averaged along the horizontal axis while the vertical axis is converted into the local twist angle. Evolution of LLs from the flat $\left(\left|\mathrm{V}_{\mathrm{Bias}}\right|<50 \mathrm{mV}\right)$ and remote bands $\left(\left|\mathrm{V}_{\text {Bias }}\right|>50 \mathrm{mV}\right)$ is clearly resolved. c-e, The same plot as (b) focusing on the evolution of the valence-band $\gamma \mathrm{LL}_{0}$ for: $\left(\mathrm{V}_{\text {Gate }}=1.7 \mathrm{~V}\right.$ (c), electron doping; $\mathrm{V}_{\text {Gate }}=0.2 \mathrm{~V}(\mathbf{d})$, near the CNP; $\mathrm{V}_{\text {Gate }}=-1.7 \mathrm{~V}(\mathbf{e})$, hole doping). Merging between $\gamma \mathrm{LL}_{0}$ and VHS occurs at higher twist angle as $\mathrm{V}_{\text {Gate }}$ is reduced. The insets in (b-e) sketch the band structure and Fermi level near $\theta=1.23^{\circ}$ and $\theta=1.15^{\circ}$. A smooth signal background is subtracted to enhance LL visibility. f-h, Point spectroscopy for $\theta=1.23^{\circ}(\mathbf{f}), 1.17^{\circ}(\mathbf{g})$, and $1.15^{\circ}(\mathbf{h})$. Black arrows in (g) and (h) indicate emerging correlated Chern phases ${ }^{910}$ after the $\gamma \mathrm{LL}_{0}$ merges with the VHS. Color coded lines show $\mathrm{V}_{\text {Gate }}$ values used in (c-e). 

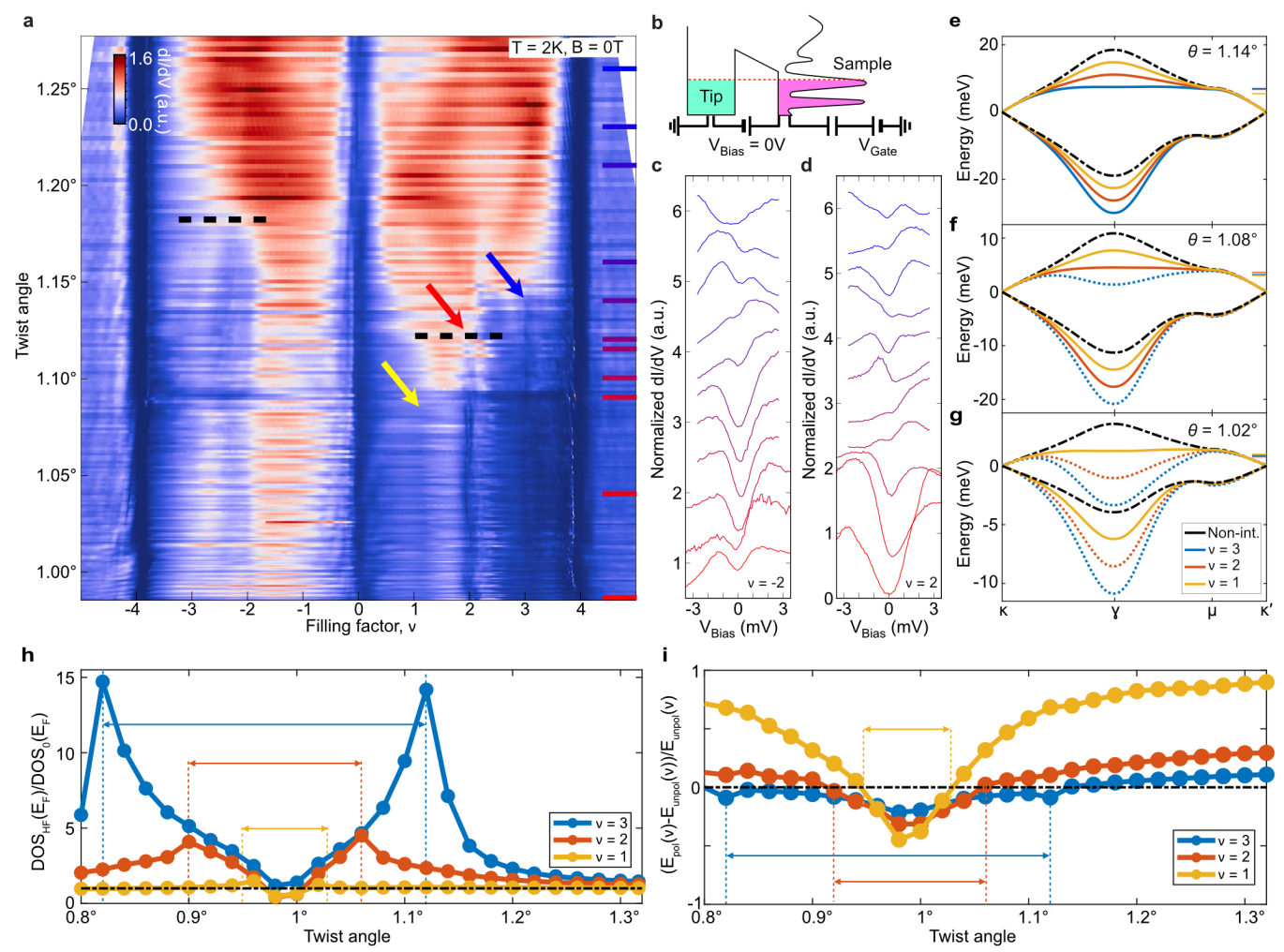

Figure 3 Emergence of zero-field correlated gaps and symmetry-breaking cascade. a, Angle and filling-factor dependence of $\mathrm{dI} / \mathrm{dV}$ near $\mathrm{E}_{\mathrm{F}}\left(\mathrm{V}_{\mathrm{Bias}}=0.3 \mathrm{mV}\right)$ at $\mathrm{B}=0 \mathrm{~T}$ taken on the same area as in Fig. 2 a,b. Correlated gaps at $\nu=-2,+1,+2,+3$, observed as an abrupt drop in $\mathrm{dI} / \mathrm{dV}$ (i.e., LDOS), develop only below certain twist angle-in contrast to regions between the flat and remote bands $(\nu= \pm 4)$ where LDOS is small for any angle. Black lines near $\nu= \pm 2$ mark the upper bound where gaps begins to emerge, while colored arrows indicate corresponding LDOS suppression regions at $\nu=+1,+2,+3$. b. Schematic showing how data in (a) is taken: $\mathrm{V}_{\text {Bias }} \approx 0$ is fixed so that the STM tip probes LDOS near the Fermi energy while $V_{\text {Gate }}$ is swept. c, d, Spectroscopy for $\nu=-2(\mathbf{c})$ and $\nu=+2(\mathbf{d})$ at different local twist angles ranging $\theta=1.26^{\circ}-0.98^{\circ}$, taken at $\mathrm{AB}$ sites. Color coding of the lines correspond to the angles marked by horizontal bars in (a). Clear correlated gaps that open only at the Fermi energy are observed only below a certain angle (small wiggles above this angle originate from trivial origins, see Supplementary Fig. S8). Each spectrum is normalized by an average $\mathrm{dI} / \mathrm{dV}$ value and offset for clarity. e-g, Interactionrenormalized band structures at different integer fillings, calculated assuming unpolarized ground states (see main text and SI, section 6). Horizontal lines indicate the relevant chemical potentials. In cases where polarized states are favorable, dotted lines are used. The non-interacting band structure is shown in black. $\mathbf{h}$, Twist-angle dependence of the DOS at $\mathrm{E}_{\mathrm{F}}$ obtained from the interaction-corrected unpolarized band structure, normalized by the non-interacting DOS. Peaks signal maximal band flattening as seen in e-g. i, Relative energy change for polarized (cascaded) states relative to unpolarized states. Interaction-driven band flattening significantly extends the range of angles, marked by arrows in (j) and (i), where this relative energy change is negative and polarization becomes energetically favourable (see SI, section 6). 

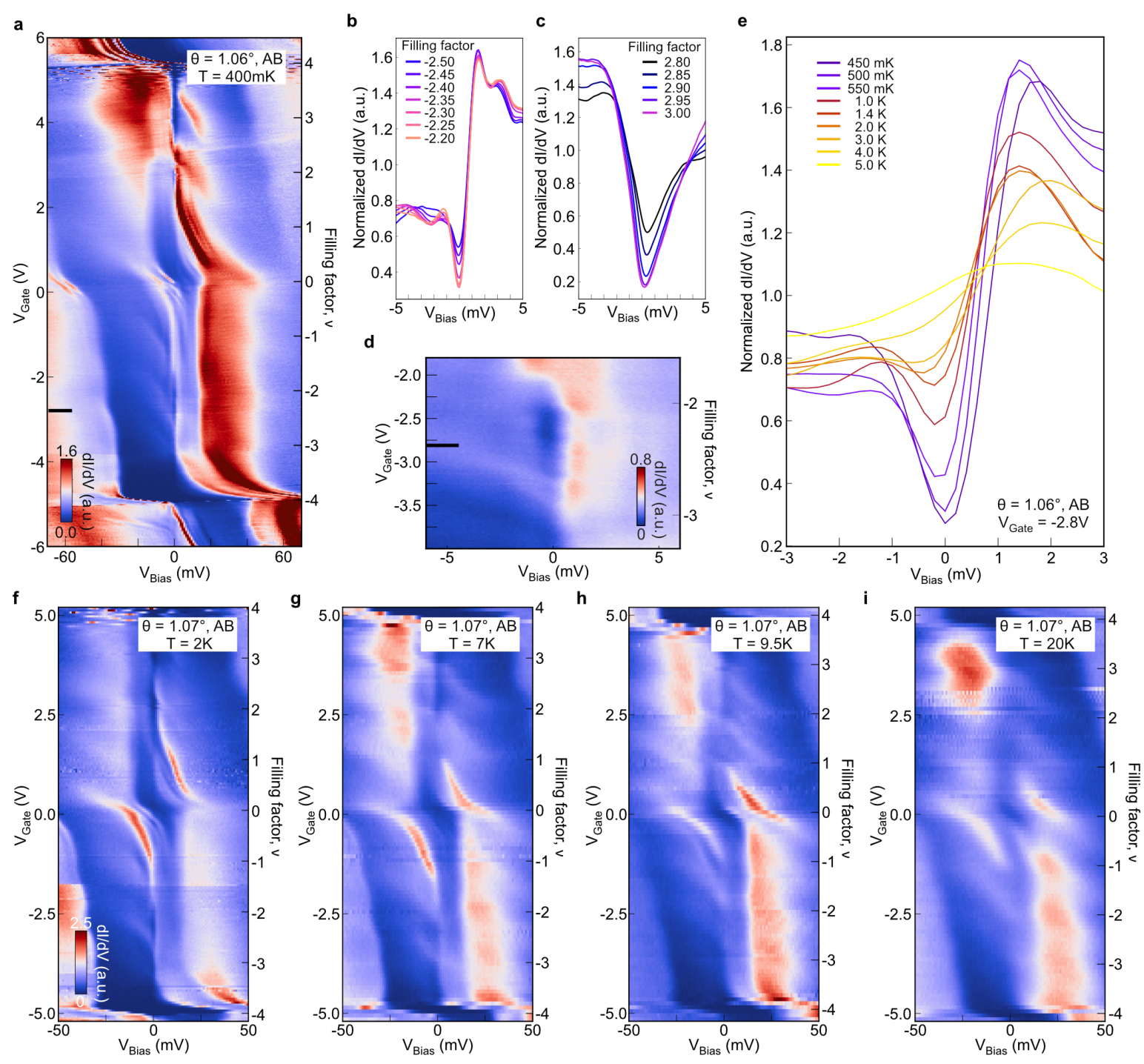

Figure 4 Temperature dependence of correlated gaps around $\nu= \pm 2$ and the symmetrybreaking cascade. a, Point spectra as a function of $\mathrm{V}_{\text {Gate }}$ for $\theta=1.06^{\circ}$ at $\mathrm{B}=0 \mathrm{~T}$. b,c, $\mathrm{dI} / \mathrm{dV}$ spectra for filling factors ranging from $\nu=-2.5$ to $\nu=-2.2$ (b) and from $\nu=2.8$ to $\nu=3.0$ (c). d, High-resolution point spectra of (a) focusing on the soft gap between $\nu=-2$ and -3 . e, $\mathrm{dI} / \mathrm{dV}$ spectra at $\mathrm{V}_{\text {Gate }}=-2.8 \mathrm{~V}$ (as indicated by black lines in $(\mathbf{a}, \mathbf{d})$ ) for temperatures ranging from $450 \mathrm{mK}$ to $5 \mathrm{~K}$ at the same tip location as (a). f-i, Point spectra as a function of $\mathrm{V}_{\text {Gate }}$ for $\theta=1.07^{\circ}$ at temperatures $\mathrm{T}=2 \mathrm{~K}(\mathbf{f}), \mathrm{T}=7 \mathrm{~K}(\mathbf{g}), \mathrm{T}=9.5 \mathrm{~K}(\mathbf{h})$, and $\mathrm{T}=20 \mathrm{~K}(\mathbf{i})$. As temperature increases, the cascade features become more pronounced, and their onset more closely follows integer filling factors, hinting at a characteristic cascade temperature scale of $\mathrm{T} \approx 20 \mathrm{~K}$ as previously noted ${ }^{28}$. 
